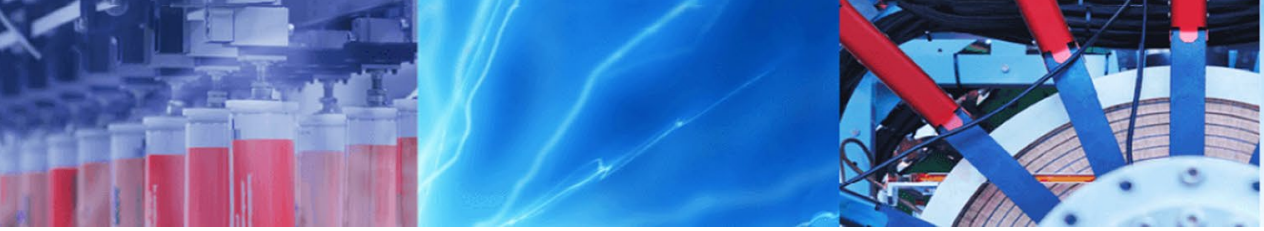

Research Article

\title{
Porosity evolution of expanded vermiculite under pressure: the effect of pre-compaction
}

\author{
Félix Balima ${ }^{1,6} \cdot$ Sylvie Le Floch $^{1} \cdot$ Alfonso San-Miguel $^{1} \cdot$ Laurence Reinert $^{2} \cdot$ Laurent Duclaux $^{2} \cdot$ An Ngoc Nguyen $^{2,7}$. \\ Isabelle Daniel ${ }^{3} \cdot$ Annie Brûlet $^{4} \cdot$ Laurent Gremillard $^{5} \cdot$ Vittoria Pischedda $^{1}$ (i)
}

(c) Springer Nature Switzerland AG 2019

\begin{abstract}
The evolution of the pore structure of compressed expanded vermiculite was investigated in situ under uniaxial stress up to 3200 bar using small-angle neutron and X-ray scattering. The studied samples have a lamellar texture in which the presence of oriented oblate pores was revealed by the anisotropic small-angle scattering patterns. The initial pore size distribution and the mechanical behaviour of the pellets at working pressure conditions are strongly related to the applied initial stresses used to pre-compact the vermiculite powder. Our study shows that the porous structure of the pellets was not modified under compression up to the pre-compaction pressure. Higher densification is associated with increasing anisotropy of the pore-matrix interface structure. The pore-matrix interface could be described with fractal geometry. The stress dependence of the system fractal dimension, apparent specific surface area and total porosity was determined and related to the meso- and macropore evolution under uniaxial stress.
\end{abstract}

Keywords Porosity $\cdot$ Vermiculite particles $\cdot$ Small-angle scattering $\cdot$ High pressure

\section{Introduction}

Vermiculite, one of the most used clay minerals, is a highcharge 2:1 phyllosilicate. The layers are composed of two sheets of silica and alumina tetrahedra (the tetrahedral sheets) joined together with an oxygen octahedral sheet containing cations such as magnesium and iron [1]. The ability to trap ions and molecules-such as water-in the interlayer space conveys appreciated exfoliation properties. Indeed, when submitted to a thermal shock, vermiculite loses its interlayer water resulting in a huge volume expansion. In its exfoliated state, vermiculite shows very interesting qualities such as low bulk density, low thermal conductivity, high melting point $\left(1240-1430{ }^{\circ} \mathrm{C}\right)$, high absorbency, high specific surface area and cation exchange properties. For all these appealing features, together with its wide availability and low cost, exfoliated vermiculite is used in many applications, as lightweight porous filler in the production of heat-insulating components [2] or as a component of sealing gaskets for hightemperature applications [3]. For specific applications, vermiculite may be manufactured by different techniques, usually including additives of different chemical nature, to obtain items of various sizes and shapes, such as pellets, sheets and films $[4,5]$.

The high porosity (up to $70 \%$ ) of the expanded vermiculite and its derivatives provides an effective way to reduce apparent density and to improve refractory property. Nevertheless, an important limitation in the application of expanded vermiculite is its ability to sustain deformation.

Sylvie Le Floch, sylvie.le-floch@univ-lyon1.fr; $₫$ Vittoria Pischedda, vittoria.pischedda@univ-lyon1.fr| ${ }^{1}$ Institut Lumière Matière, UMR5306 Université Lyon 1-CNRS, Université de Lyon, 69622 Villeurbanne, France. ${ }^{2}$ LCME, Univ. Savoie Mont Blanc, 73000 Chambéry, France. ${ }^{3}$ Laboratoire de Géologie de Lyon, UMR 5276 CNRS - Université Lyon 1 - ENS de Lyon, Université de Lyon, 69622 Villeurbanne, France. ${ }^{4}$ Laboratoire Léon Brillouin, UMR12, CEA-CNRS, CEA-Saclay, 91191 Gif-sur-Yvette, France. ${ }^{5}$ Mateis UMR5510 CNRS, Université de Lyon, INSA-LYON, 69621 Villeurbanne, France. ${ }^{6}$ Present Address: Institut de Chimie de la Matière Condensée de Bordeaux (ICMCB)-CNRS, 33608 Pessac Cedex, France. ${ }^{7}$ Present Address: University of Science, Ho Chi Minh City, Viet Nam.

SN Applied Sciences (2019) 1:629| https://doi.org/10.1007/s42452-019-0627-9 
Many applications include the compaction processes of expanded vermiculite or its integration in composite materials. The mechanical properties as well as the apparent density of vermiculite are linked to the particle size [6]. Understanding the relationship between porosity, particle size, mechanical properties and thermal insulating capacity is fundamental for the design of new refractory materials for a wide range of important technological fields such as furnaces, reactors, building, petrochemical and environmental applications.

Porosity of clays has been already studied using different methods such as mercury intrusion porosimetry [7, 8], electron microscopy [9] or small-angle scattering (SAS) [10-13]. The use of SAS for porous material investigation has been established as a non-invasive method and appropriate to investigate both open and closed porosities.

The previous studies of compacted layered silicates have described the evolution of the porosity as a function of the uniaxial compression derived from the interpretation of the stress-strain curves and ex situ X-ray diffraction or small-angle neutron scattering (SANS) analysis $[12,14]$.

In recent works $[15,16]$, we have shown that in situ high-pressure SAS is a powerful method to understand the evolution of the meso- and macropore structure as a function of the working stress in lamellar systems such as expanded graphite. In this paper, we consider vermiculite pellets that, when compressed in the $350-800$ bar range, present very good mechanical and sealing properties suitable for industrial use [19]. By high pressure in situ smallangle scattering study, combined with ex situ porosity characterisation methods, we aim to better understand the effect of porosity variation on the mechanical response of these compressed vermiculite pellets.

\section{Materials and methods}

\subsection{Samples and high-pressure cells}

The vermiculite powder is composed of platelet-like particles in the $0.5-20 \mu \mathrm{m}$ range $\left(d_{50}=1.5 \mu \mathrm{m}\right.$ and $\left.d_{90}=7 \mu \mathrm{m}\right)$ with a nanometric thickness $(8 \mathrm{~nm})$, resulting from the sonication in a $35 \mathrm{wt} \%$ hydrogen peroxide solution ( $20 \mathrm{kHz}, 350 \mathrm{~W}, 5 \mathrm{~h}$, solid/liquid weight ratio equal to $1 \%$ ) of potassium saturated millimetric vermiculite flakes ( $<2 \mathrm{~mm}$, exfoliated "Granutec ${ }^{\circ} \mathrm{E}$ ", fine grade, Yuli, China). The average chemical composition of the half lattice cell calculated from elemental analysis is $\left(\mathrm{Si}_{3} \mathrm{Al}\right) \mathrm{Mg}_{2.62} \mathrm{Fe}_{0.32} \mathrm{~T}$ $\mathrm{i}_{0.06} \mathrm{O}_{10}(\mathrm{OH})_{2} \mathrm{~K}_{0.61}$. More details on the sample and on the sonication procedure were given in the previous works [17, 18]. The samples used for the SAS experiments were made by pre-compacting the vermiculite powder at a pressure $P_{c}=350$ bar or 800 bar, resulting in two different pellet densities. These $P_{\mathrm{c}}$ values have been chosen taking into account the results of a previous study on the sealing and mechanical properties of vermiculite gaskets [19]. In use, the gasket has to be compressed at a minimum clamping pressure of 350 bar in order to adopt the shape of the sealing support and minimise the leak at the interface. The manufactured gasket must also accommodate the weak motions of the sealing support during its life cycle. Thus, elastic mechanical properties are required. The best sealing and mechanical properties have been obtained for a gasket formed under 800 bar.

The porosity of the pellets was measured by mercury porosimetry using a Micromeritics AutoPore IV 9400 Series instrument. About $1.4 \mathrm{~g}$ of sample was degassed in the porosimeter to $50 \mu \mathrm{m} \mathrm{Hg}$ before the mercury intrusion. The intrusion was performed in the pressure range of 0.035 to 4000 bar, allowing the penetration of the pores of diameter ranging between $350 \mu \mathrm{m}$ and $3 \mathrm{~nm}$, with an accuracy of about $0.25 \%$. Based on the assumption of cylindrical pores, the pore size distribution was calculated by the Washburn equation: $D=(-4 \sigma \cos \Theta) / P$, where $P$ is the absolute injection pressure $(\mathrm{Pa}), D$ is the pore diameter $(\mathrm{m})$ when mercury enters at the pressure $P, \Theta$ is the contact angle between mercury and the pore surface (assumed to be $130^{\circ}$ in the experiments) and $\sigma$ is the interfacial tension of mercury (set to $0.485 \mathrm{~J} \mathrm{~m}^{-2}$ ). The bulk densities were calculated from mercury intrusion analyses after determination of the volume of a weighted sample which was assumed to be equal to the difference between the volume of the empty penetrometer and the volume of the mercury intruded at low pressure. The skeletal density, determined by the ratio of the apparent volume subtracted from its measured open porosity to its mass, is $2.7 \mathrm{~g} \mathrm{~cm}^{-3}$.

The samples used for SANS were pre-compacted in a 19-mm-long, 18-mm-high and 2-mm-thick sample chamber, inside an in-house designed high-pressure cell (Fig. 1a) [20]. The samples used for the small-angle $X$-ray scattering (SAXS) investigations were pre-compacted in a $700 \mu \mathrm{m}$ diameter hole drilled in a 300- $\mu \mathrm{m}$-thick nickel gasket in a diamond anvil cell (DAC) (Fig. 1b). More details on these high-pressure cells used for SAS collections can be found in ref [20]. Hereafter, V800 and V350 are referred to the higher-density pellet $\left(P_{c}=800\right.$ bar) and the lower-density one $\left(P_{\mathrm{c}}=350 \mathrm{bar}\right)$, respectively. The same cells were used during the in situ SANS and SAXS measurements; the applied uniaxial pressure had the same out-of-plane direction as the pre-compaction stress (Fig. 1).

\subsection{Small-angle scattering experiments}

SANS experiments were performed on PAXE and TPA beamlines at Laboratoire Léon Brillouin (Saclay, France) 


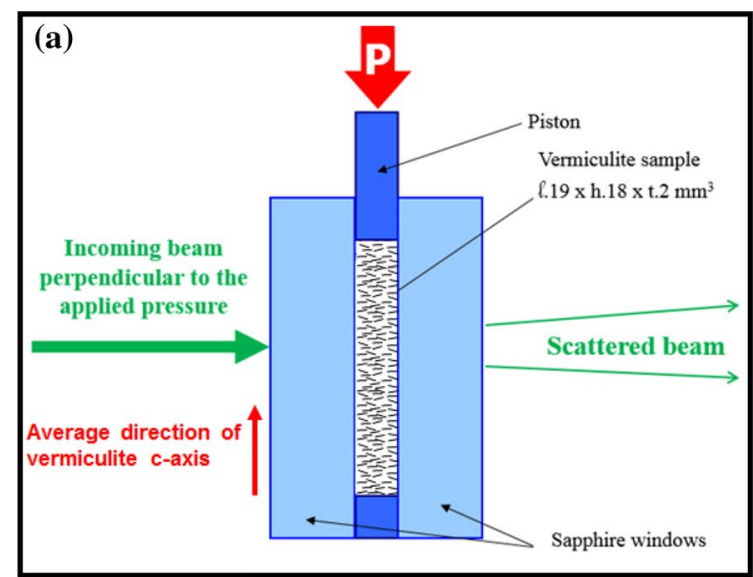

Fig. 1 Schematic view of the high-pressure experiment setups. a The high-pressure cell used for the SANS experiment [20]. The vermiculite sample had $I=19 \mathrm{~mm}$ length, $t=2 \mathrm{~mm}$ thickness and $h=18 \mathrm{~mm}$ height. The incoming neutron beam was perpendicular to the applied pressure and to the average direction of the c-axis of vermiculite. $\mathbf{b}$ The diamond anvil cell used for the SAXS experiment: the vermiculite sample had a $t=300 \mu \mathrm{m}$ thickness and a $d=700 \mu \mathrm{m}$

using a $6 \AA$ wavelength and a $7 \mathrm{~mm}$ neutron beam size. The data collected on the $\mathrm{BF}_{3}$ detector on PAXE were merged with those collected on the MAR image plate high-resolution detector on TPA. By merging data on the $\left[5 * 10^{-4}-0.75 * 10^{-2}\right] \AA^{-1}$ and $\left[5 * 10^{-2}-0.18\right] \AA^{-1}$ ranges of TPA and PAXE, respectively, we were able to cover a broad q range: $5^{*} 10^{-4}<q<0.18 \AA^{-1}$. Raw data were corrected from the sample transmission and the empty cell and background scattering. The absolute intensity (in $\mathrm{cm}^{-1}$ units) was obtained by using plexiglass as standard. For the in situ pressure studies, an in-house designed pressure cell was used [20]. The compression chamber was limited by two 4-mm-thick sapphire windows allowing a full spectroscopic access to the beam. A piston connected to a hydraulic pump generated an adjustable pressure up to 180 bar corresponding to a 1500 bar pressure applied on the sample. The incoming neutron beam was perpendicular to the applied pressure direction and parallel to the preferential in-plane direction of the vermiculite plateletlike particles (Fig. 1a).

The SAXS experiment was performed on the SWING beamline at synchrotron SOLEIL (Saint Aubin, France) with a $15 \mathrm{keV}$ energy and $400 \mu \mathrm{m} / 300 \mu \mathrm{m}$ beam size using a PCCD170170 (AVIEX) two-dimensional detector. Using a fixed detector-sample distance of $6 \mathrm{~m}$, the scattered intensity was measured in a $q$ momentum transfer range of $1.7^{*} 10^{-3}<q<0.1 \AA^{-1}$. The intensity (in arbitrary units) was corrected from the empty cell and background scattering. The in situ study under pressure was carried out up to 3200 bar by using a diamond anvil cell especially adapted to lower-pressure experiments [21]. The ruby fluorescence

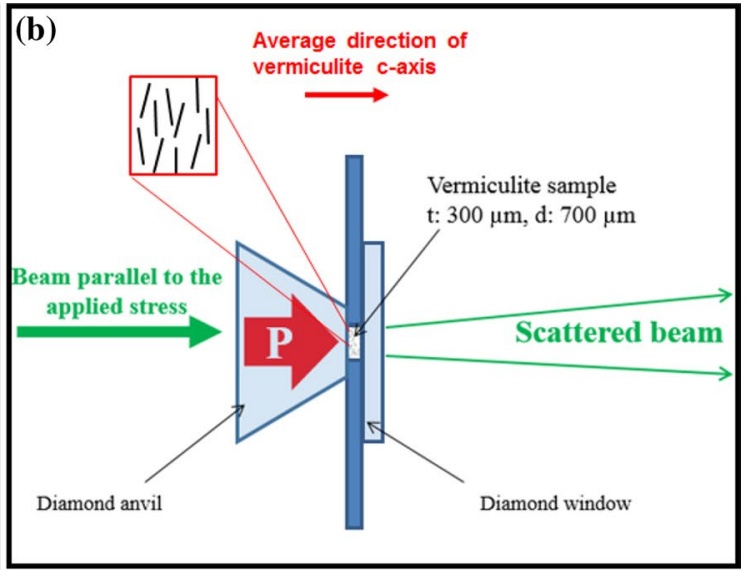

diameter. The incoming X-ray beam was parallel to the applied pressure and to the average direction of the $c$-axis of vermiculite. The inset shows a magnification of a schematic view of the vermiculite crystallite orientation. Note the opposite orientation of the sample $c$-axis with respect to the beam in the two setups. In both in situ SANS and SAXS experiments under pressure, samples were pre-formed with a compaction pressure $\left(P_{\mathrm{c}}\right)$ of 800 or 350 bar

was used as a pressure calibrant. The total thickness of the diamond crossed by the X-ray beam was $2.8 \mathrm{~mm}$. The incoming beam was parallel to the applied stress direction (Fig. 1b).

The SANS-integrated spectra were analysed by using the LLB in-house software PAsiNET and written scripts. The SAXS data were processed by using written scripts.

Porous media have been very often studied through the characterisation of their interface irregularities [12, 22-24]. When the interface is scale invariant within at least one order of magnitude, a fractal approach can be used [25]. The correlation function is then given by $g(r) \propto r^{3-d}$ [22] where $r$ is the spatial variable and $d$ the fractal dimension of the system. Theoretical fractal systems should have unlimited scale invariance, but the real fractal behaviour of a system is limited within a $q$ range which has to be specified [25]. The Fourier transform of $g(r)$ gives the scattered intensity: $I(q)$.

In the high $q$ region ( $q r \gg 1$ ), the scattering is dominated by the interface contribution and the scattered intensity follows a power law $I(q) \propto q^{-(6-d)}$. For a smooth interface $d=2$, while for a very rough surface it approaches 3 . The fractal dimension can then be determined from the slope of Logl versus Logq.

The specific surface $S$ (in $\mathrm{m}^{2} \mathrm{~cm}^{3}$ units) can be extracted from the asymptotic value of $I(q) q^{\alpha}(\alpha=6-d)$ at high $q$ values. It is given by:

$S=\sigma \times r_{0}^{2-d}$,

where $r_{0}$ is a length scale and $\sigma$ is proportional to the high $q$ limit of $I(q)$ according to the formula: 
$\sigma=\frac{\lim _{q \rightarrow \infty} I(q) q^{(6-d)}}{\pi(\Delta \rho)^{2} F(d)}$

where $\Delta \rho$ is the scattering length density contrast between pore and matrix and $F(d)$ the function defined by Hurd et al. [25].

The Debye model was used to extract the $\xi$ correlation length in the low $q$ range for a random two-phase system. The correlation lengths were then obtained by fitting the equation: $I(q)=I_{0}\left(1+q^{2} \xi^{2}\right)^{2}$ to the experimental data in a graph $I(q)^{-1 / 2}$ versus $q^{2}$.

\section{Results and discussion}

\subsection{Ex situ porosity characterisation}

The evolution of the mesoporous $(2<\varnothing<50 \mathrm{~nm})$ and macroporous $(\varnothing>50 \mathrm{~nm}$ ) volumes of the V 350 and V800 pellets was analysed by mercury intrusion porosimetry before and after the pressure cycle up to 1200 bar (Fig. 2). Mercury intrusion, which assumes cylindrical pore morphology, allows probing the open porosity. V350, precompacted at 350 bar, possesses a bimodal distribution of the pore size (around 200 and $40 \mathrm{~nm}$ ) and a total open porosity of $43 \%$ (Fig. 2a). Pressing V350 at 1200 bar led to a decrease in the macroporous and an increase in the mesoporous volumes. The total open porosity decreased to $38 \%$, and the bimodal distribution of the pore diameter shifted to 130 and $20 \mathrm{~nm}$.

V800 pore size distribution revealed a narrower bimodal distribution of the pore diameter (120 and $50 \mathrm{~nm}$ ) than the V350 sample. After a pressure cycle up to 1200 bar, the mesoporous volume increased with a shift of its average pore diameter to $20 \mathrm{~nm}$ while the macroporous volume decreased with unchanged average porous size. The concomitant variations of meso- and macropore volumes could explain the almost invariant total porosity observed

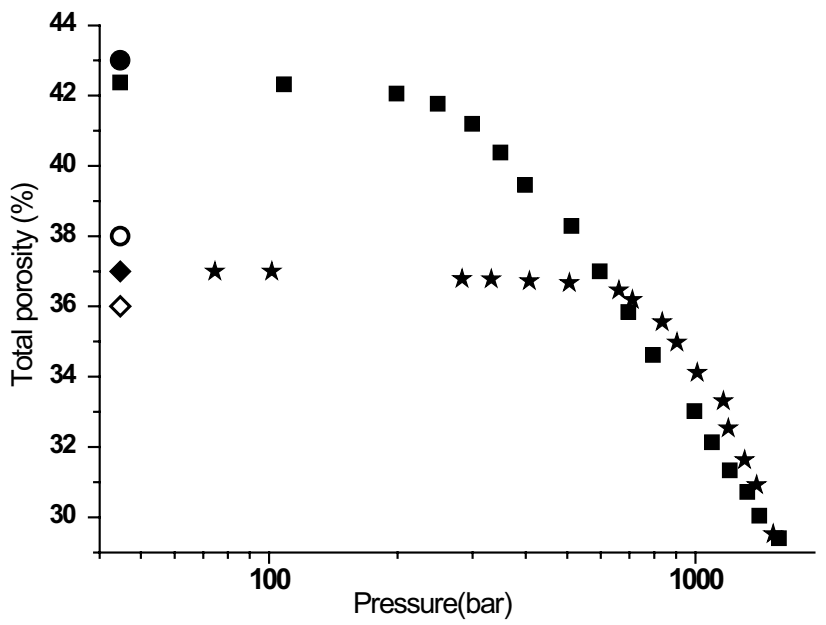

Fig. 3 Pressure dependence of the total porosity for V350 (squares) and V800 (stars) sample calculated from the sample volume variation versus applied pressure. For comparison, mean total porosity, according to mercury intrusion, is indicated with circles and rhombus for the V350 and V800, respectively. Values for quenched samples after compression to 1200 bar are indicated with empty symbols

(from $37 \%$ before the pressure cycle to $36 \%$ after depressurizing from $1200 \mathrm{bar}$ ) in this sample.

As shown in Fig. 2, the final pore size distribution after depressurization from 1200 bar is independent of the initial precompaction pressure (350 or 800 bar): the two discontinuous profiles in Fig. $2 \mathrm{a}, \mathrm{b}$ are identical within error bars.

\subsection{In situ porosity characterisation}

The pressure cell piston displacement was measured during the SANS high-pressure experiment. This allowed determining the sample volume variation and consequently the total porosity as a function of the applied pressure (Fig. 3). The decompression curves were not recorded because the relaxation of the piston was limited by the friction between the piston and the sample
Fig. 2 Pore size distribution curves determined by mercury intrusion for the a V350 sample and $\mathbf{b}$ V800 sample before compression (solid lines) and after being decompressed from 1200 bar (dashed lines)
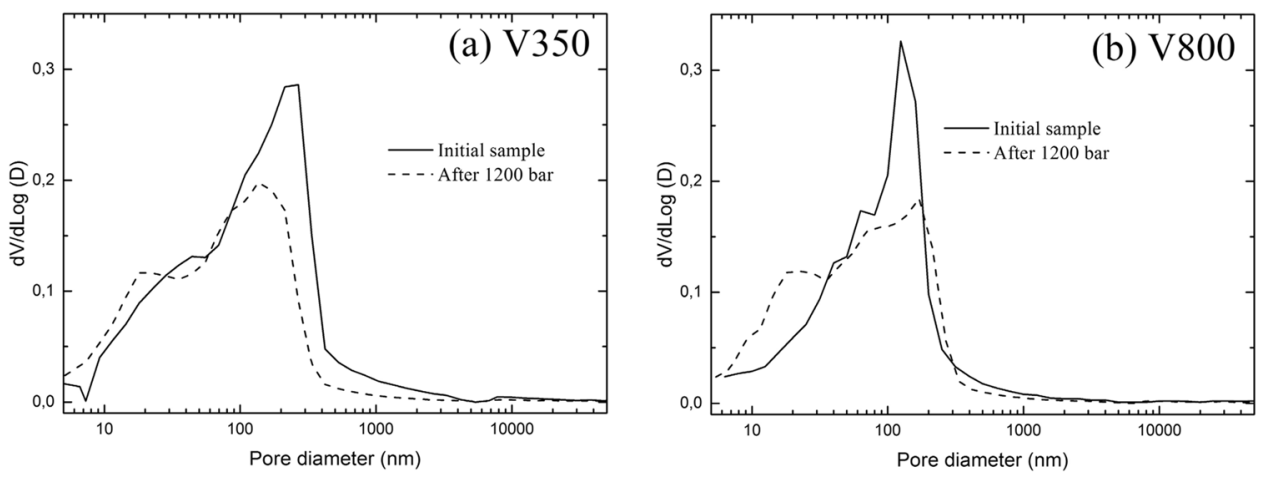
chamber. The semi-logarithmic plot of the compression curves obtained for the V350 and V800 samples shows two distinct regions: a plateau parallel to the $x$-axis and the second regime showing a decrease in porosity as increasing the applied pressure. For both samples, the transition between these two regimes corresponds to $P_{\mathrm{c}}-100 \mathrm{bar}$ (i.e. around 250 bar and 700 bar for V350 and V800 sample, respectively). Up to $P_{c}-100$ bar, the total porosity remains essentially stable. As the stress exceeds $P_{c}-100$ bar, the total porosity decreases. This compressive behaviour is similar to that observed for soils [14]. As in natural compacted clays, the compressive properties of our samples are mainly determined by the initial void ratio which depends on the compaction pressure value $P_{c}$.

The porosity of $\mathrm{V} 350$ reaches the value of $\sim 37 \%$ when compressed at $\sim 600$ bar. This value corresponds to the starting porosity of the pre-compressed V800. According to mercury intrusion, the mean total porosity of the quenched sample after compression to 1200 bar is $38 \%$ and $36 \%$ for $\mathrm{V} 350$ and $\mathrm{V} 800$, respectively. These results indicate that: (1) vermiculite pellets have mostly open porosity; (2) the compression between 700 and 1200 bar is mainly reversible suggesting an elastic behaviour of the pellets compacted to at least $700-800$ bar.

The small-angle scattering data were further analysed in order to better understand the evolution during compression and the influence of the porosity on the mechanical properties of the pre-compacted expanded vermiculite.

Platelet-like vermiculite particles are known to orient preferentially along the direction perpendicular to an applied force [26] or through sedimentation [27].

The small-angle scattered pattern shape depends on the orientation of the incoming beam with respect to the preferential orientation of the vermiculite particles. As the incoming beam was perpendicular to this preferential orientation, the scattered patterns were isotropic. This was observed for the in situ SAXS experiments under pressure (Fig. 4a). On the other hand, in the case of incoming beam parallel to the crystallite preferential orientation, the resulting patterns were elliptic. This was observed for the in situ SANS experiments under pressure (Fig. 4b). Combining the in-plane and out-of-plane scattered patterns in ambient conditions, the average pore shape can be modelled by a spheroid (Fig. 4c). This model explains why, due to the different sample orientation, SAXS experiments show isotropic scattering and SANS experiments do not. The proposed pore shape allowed modelling the mean pore morphology compatible with the observed patterns. To complete this model, we need to keep in mind that as mercury porosimetry intrusion results suggest: (1) the pore structure is polydispersive in size as the pore dimension involves two scales (Fig. 2): mesopore $(2-50 \mathrm{~nm})$ and macropore (>50 nm); (2) the oblate voids are interconnected. However, the low-structured signal of the obtained SANS spectra did not allow us to fit them using more sophisticated models as hierarchically structured ones $[28,29]$. Even if such average model constitutes a crude simplification, it agrees well with experimental data and it will be used to help to understand our results.

As the studied systems are anisotropic, the different average parameters were obtained from the SANS experimental data along two perpendicular directions [15] using a $30^{\circ}$ angular sector average method [24] (for more details see also our previous works $[15,16])$. The subscripted symbols //or $\perp$ refer to data collected along the direction parallel or perpendicular to the applied pressure direction,
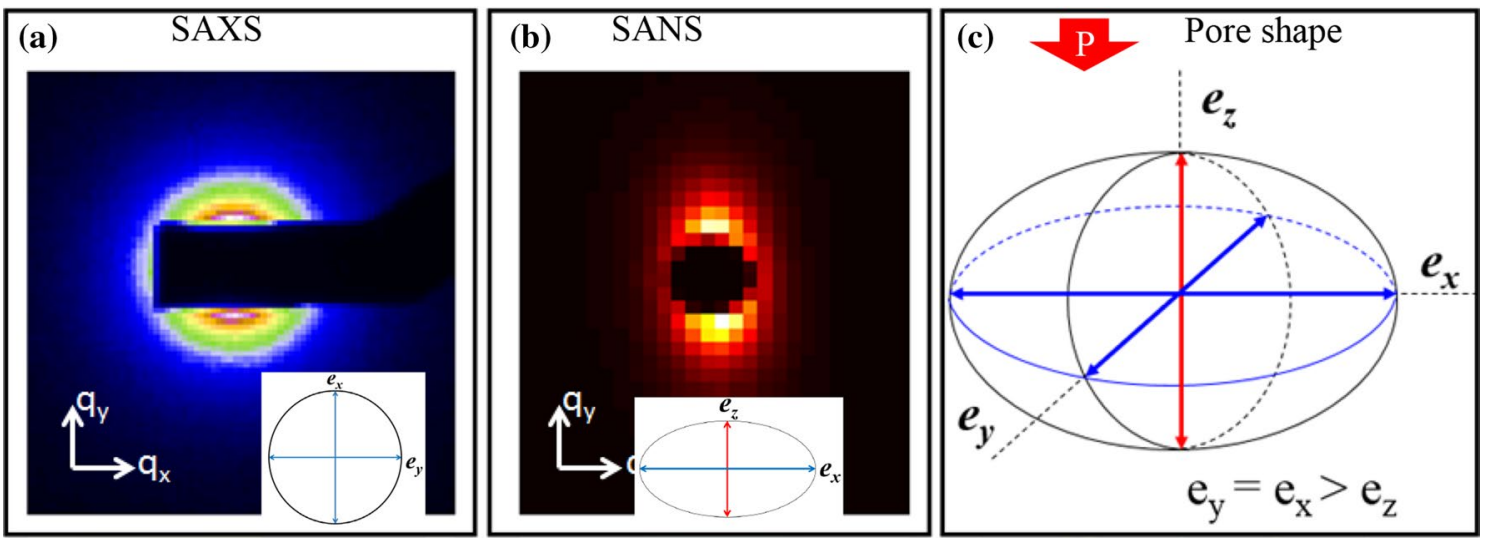

Fig. 4 Patterns collected at ambient pressure for the V800 sample. a Scattered patterns obtained by in situ SAXS experiments were isotropic as in this configuration the incoming beam was normal to the in-plane direction of the vermiculite platelet-like particles. $\mathbf{b}$ The scattered patterns were elliptical from in situ SANS where the incoming beam was parallel to the in-plane direction of the ver- miculite particles. In both cases, pressure was applied along the out-of-plane direction according to the $e_{\mathrm{z}}$ axis. c Schematic oblate spheroid representing the pore morphology deduced from the symmetry of the SANS and SAXS signal. The experimental evidence of a spheroidal pore shape is established for all the studied samples 
respectively. SANS-integrated intensities versus q along the axes perpendicular and parallel to the applied pressure of the V350 are shown in Fig. 5.

The low $q$ region is dominated by the scatterer form factor. As the studied system is not random, the Debye model was used by introducing a correlation length, a characteristic parameter of the system, along each of the two axes of the elliptic pattern $[12,15,30]$. From the linear slope of $I(q)^{-1 / 2}$ versus $q^{2}$ plot, the correlation length was estimated parallel $\left(\xi_{/ \mid}\right)$and perpendicular $\left(\xi_{\perp}\right)$ to the applied pressure and plotted in Fig. 6 . The estimated values at ambient pressure for $V 350$ are $\xi_{/ /}=36.4 \mathrm{~nm}$ and $\xi_{\perp}=53.5 \mathrm{~nm}$ and $\xi_{/ /}=33.3 \mathrm{~nm}$ and $\xi_{\perp}=56.3 \mathrm{~nm}$ for V800. V350 has a lower mean pore aspect ratio compared to $\mathrm{V} 800$. This can be related to the different motions and rearrangements of platelet-like particles under higher compression that results in flattening the pore ellipsoid. Within the error bars, the correlation lengths slightly decrease with increasing pressure for the V350 sample, while for the V800 pellet they are almost non-sensitive to further applied pressure higher than the compaction one (Fig. 6). Though these values do not vary much under compression, they are consistent with the spheroidal average pore model for both samples at ambient conditions and under compression.

In the high $q$ region, the scattered intensity exhibits a linear region for more than a decade (Fig. 5). This region follows the power law $I(q) \propto q^{-(6-d)}$ where $2<d<3$. Such deviation of the Porod's law reveals the fractal behaviour of our samples as stated above.

The evolution of the $d$ fractal dimension as a function of the applied pressure was calculated from a Log/ versus $\log q$ fit in the linear region. Figure 7 displays the fractal dimension evolution under pressure for V350 and V800 samples where $d_{\perp}$ and $d_{/ /}$are the fractal dimensions computed from the SANS data following the directions perpendicular and parallel to the applied pressure. The fractal dimension $d$ was also extracted from the SAXS isotropic patterns for the V350 sample up to 3200 bar. The fractal dimension gives information on the interface evolution under pressure in the considered pore size range.

For the V350 sample, considering the two different high-pressure experimental configurations used (Fig. 1a), the SAXS isotropic $d$ fractal dimension value is comparable to $d_{\perp}$ value from the SANS experiment.

The $d_{/ /}$and $d_{\perp}$ initial fractal dimensions are similar for the V350 sample; this reveals a homogenous structure of the pore-matrix interface within incertitude. On the other hand, for the highly pre-compacted V800 sample, the initial values of $d_{\perp}$ and $d_{/ /}$differ significantly. Our observation points out that higher densification is associated with increasing anisotropy of the pore-matrix interface structure. Interface appears rougher along the $d_{/ /}$direction.
Fig. 5 SANS-integrated intensities versus $q$ along the axes perpendicular (a) and parallel (b) to the applied pressure of the V350 sample at different pressures. The data collected on the $\mathrm{BF}_{3}$ detector on PAXE were merged with those collected on the MAR image plate high-resolution detector on TPA beamline
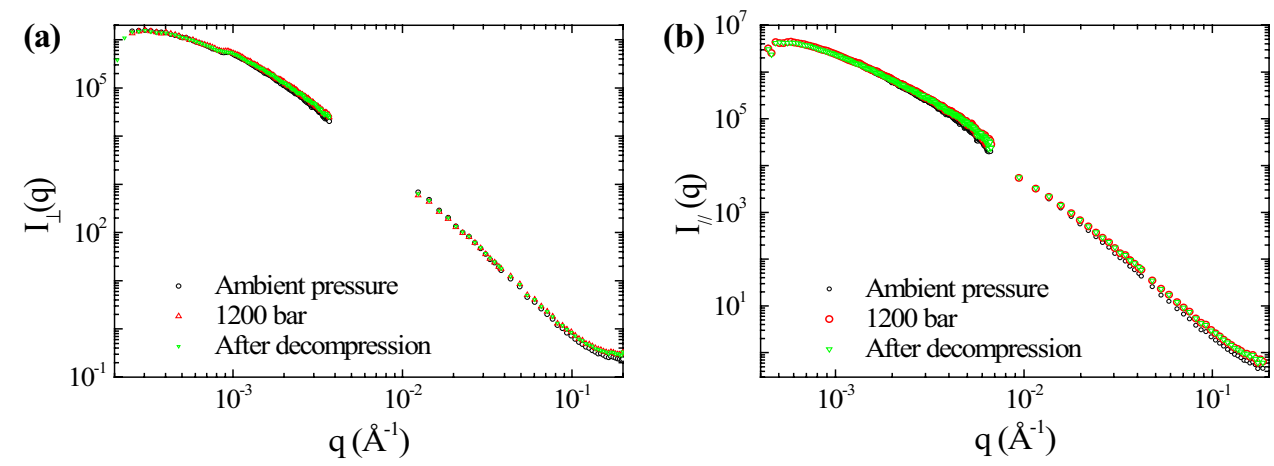

Fig. 6 Evolution of the correlation length with pressure for V350 (a) and V800 (b) samples obtained from SANS. Lines are visual guides
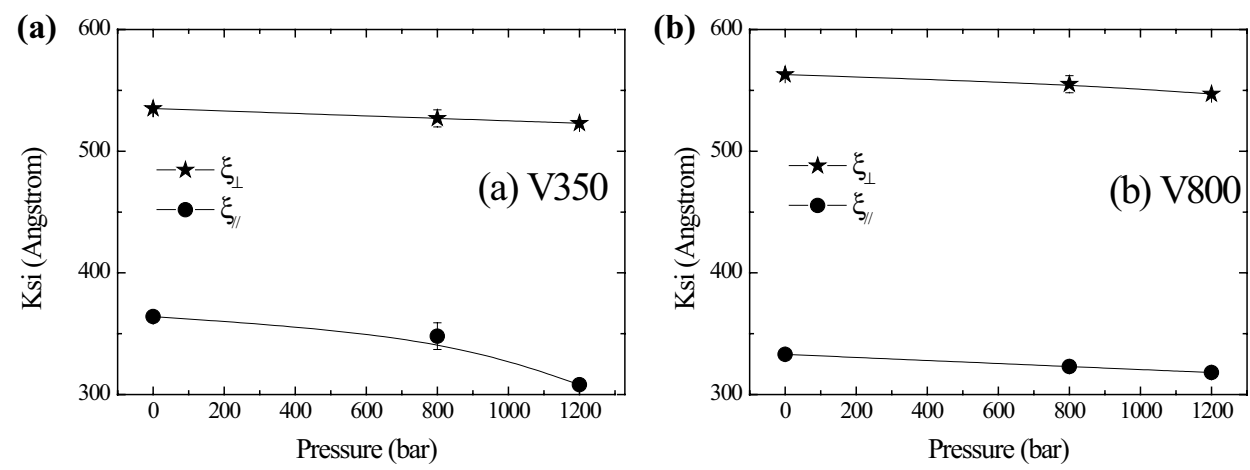
Fig. 7 Fractal dimension evolution versus applied pressure for V350 (a) and V800 (b) obtained from SANS (in black) and SAXS (in blue) data. Filled symbols stand for compression, and empty symbols for decompression. SAXS experiment was performed on the V350 sample up to 3200 bar. Lines are visual guides
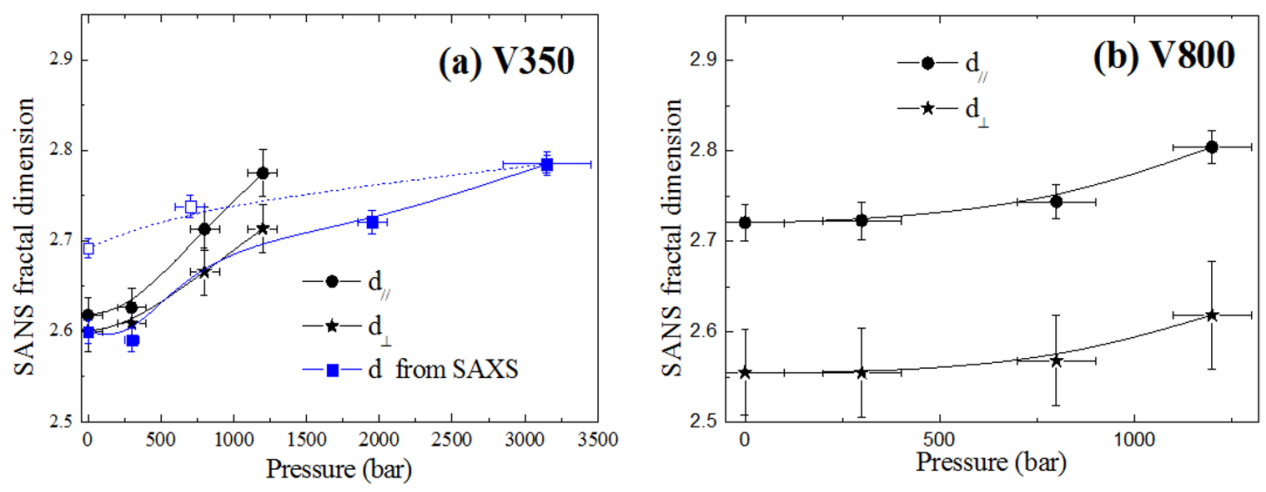

In both V350 and V800, the in situ variation of the fractal dimension as a function of applied pressure starts only above a critical value that corresponds to the compaction pressure values, $P_{\mathrm{c}}$. Above $P_{c}$, the fractal dimension increases in both V350 and V800 up to the maximum experimental pressure (1200 bar for SANS experiments and 3200 bar for SAXS experiments). The fractal dimension increase is related to the induced texture modification leading to a rougher interface between the matrix and the pore. After decompression from 3200 bar, the value of the SAXS fractal dimension of $\mathrm{V} 350$, back to ambient pressure, is lower than the one reached at 2000 bar, thus confirming the elastic behaviour of vermiculite pellets after being compressed at a sufficient pressure, not determined in this work, but comprised between 800 and 2000 bar.

The particles or particle aggregates rearrangement should likely yield to an evolution of the specific surface area. In fractal systems, this parameter is related to the fractal evolution and could lead to an accurate understanding of the texture evolution under pressure $[15,16]$.

The in situ evolution of the specific surface as a function of the applied stress can be observed in Fig. 8 where $\mathrm{AS}_{/ /}$and $\mathrm{AS}_{\perp}$ are the SANS apparent specific surface areas parallel and perpendicular to the applied pressure, respectively.

The initial values of $A S_{/ /}$and $A S_{\perp}$ evidence a certain anisotropy for both samples which are accentuated with compaction pressure. The initial values of $\mathrm{AS}_{/ /}$and $\mathrm{AS}_{\perp}$ in the $\mathrm{V} 800$ samples are close to the values reached for $\mathrm{V} 350$ sample at 800 bar.

The apparent specific surface area variation with applied pressure is almost negligible up to a pressure close to $P_{c}$. Similarly to the fractal dimension behaviour under stress, the modification of the apparent specific surface area starts when the applied pressure gets close to the consolidation pressure value. Above $P_{c^{\prime}}$ in the SANS experiment, the $\mathrm{AS}_{\text {// }}$ apparent specific surface area increases while $\mathrm{AS}_{\perp}$ is almost constant.

\section{Conclusions}

Pre-compressed expanded potassic vermiculite samples, used as main components of high-temperature-resistant sealing gaskets, were investigated by using in situ smallangle scattering study under uniaxial stress in order to elucidate the relationship between their mechanical behaviour under compression and the porosity. Combining ex situ and in situ porosity characterisation techniques, the manufacturing stress conditions of vermiculite powders have been related to the operating mechanical behaviour under stress. By sector analysis of the SANS anisotropic data, the porosity variation and particle behaviour were investigated in parallel and perpendicularly to the
Fig. 8 Apparent specific surface area evolution versus the applied pressure derived from SANS data. Lines are visual guides
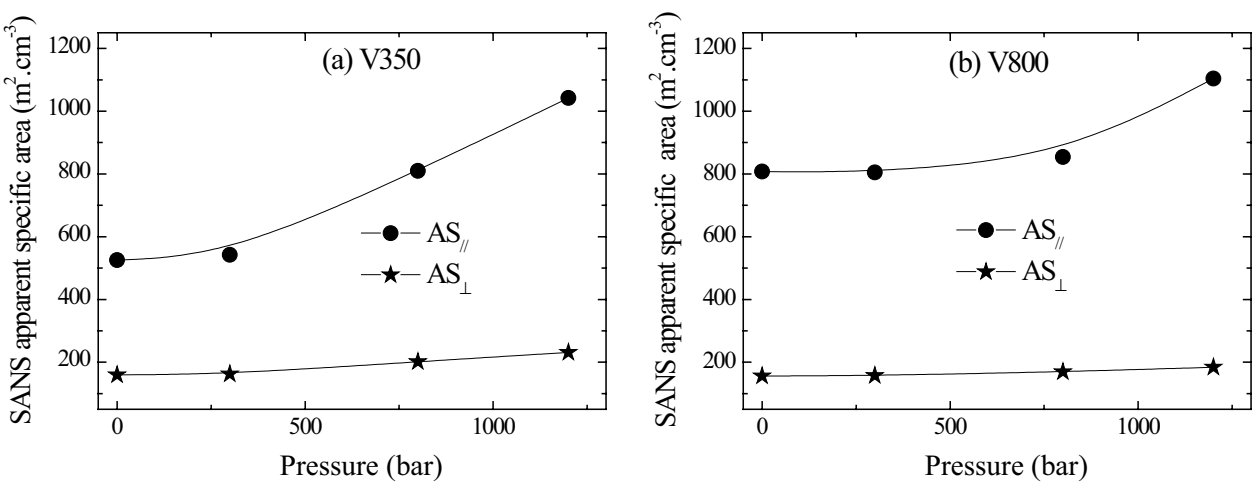

SN Applied Sciences A SPRINGER NATURE journal 
platelet-like particle preferential orientation. Such characterisation has revealed that the pores can be described by a spheroid-like morphology that flattens under compression up to 3200 bar.

Examining together, all the results important conclusions can be drawn: (1) the pore structure and the mechanical behaviour of the pellets are not modified under compression from ambient pressure up to $P_{\mathrm{c}}-100 \mathrm{bar}$. $P_{\mathrm{c}^{\prime}}$ the pre-compaction pressure, is equal to 350 or 800 bar in our case. The behaviour of clays had already been found to depend on their consolidation pressure values [27]. The vermiculite crystallite reorientation during the consolidation process results probably in straightening and hardening the sample. This irreversible behaviour with rearrangement and increasing interlocking of crystallites and consequent irreversible reduction of pore volume occurs up to 800 bar. (2) Above 800 bar, the decrease in the porosity volume observed for $\mathrm{V} 350$ and V800 is reversible (Fig. 3), but the change of the pore size distribution (Fig. 2), for the sample compressed at 1200 bar, evidences that the topology of the pore network changed irreversibly between 800 and 1200 bar. This is also evidenced by the rise of the fractal dimension (Fig. 7) that characterises an increase in the surface roughness of the pore-matrix interface. This roughness can be explained by the formation of bottlenecks in the porous network that can explain also the different pore size distributions observed by mercury porosimetry measurements for the samples compressed at 1200 bar. The absence of closed porosity formation and the constant specific surface area upon compression along the direction perpendicular to the applied pressure (Fig. 8) go against the hypothesis of a division of the pores. The involved mechanism does not look similar to the one previously described for expanded graphite [15] which involves instead the collapse and subsequent splitting of the pores into smaller size pores. (3) From 2000 to 3200 bar (the highest pressure reached in the SAXS experiment for V350 sample), a reversible slight increase in the fractal dimension is observed. Compression above 2000 bar has the effect to induce a reversible elastic behaviour of the flexible crystallites.

Acknowledgements This study was funded by the Agence Nationale de la Recherche (ANR) through MATETPRO project ANR-08MAPR-0011. We are very grateful to Frank Legrand for his efficient implication in the high-pressure cell development, Florian Meneau for his help during SAXS collection data and Hervé Cardon for his assistance during the diamond anvil cell preparation. We thank the synchrotron SOLEIL (France) for time allocation at SWING beamline and for the use of the high-pressure laboratory.

\section{Compliance with ethical standards}

Conflict of interest All authors declare that there is no conflict of interest.

\section{References}

1. Bergaya F, Lagaly G (2013) Handbook of clay science, 2 nd edn. Elsevier, Amsterdam

2. El Mouzdahir Y, Elmchaouri A, Mahboub R et al (2009) Synthesis of nano-layered vermiculite of low density by thermal treatment. Powder Technol 189:2-5. https://doi.org/10.1016/j. powtec.2008.06.013

3. Hoyes JR, Woolfenden S (2008) Process for the production of vermiculite foil, US7321009 B2

4. Minker-Villemin C, Bowen P, Lemaître J, Ring TA (1998) Preparation and properties of potassium-vermiculite films. J Mater Res 13:228-236. https://doi.org/10.1557/JMR.1998.0031

5. McAloon KT, Heeson IE, Cleaver GE (1985) Production of vermiculite products, US4539046

6. Suvorov SA, Skurikhin VV (2003) Vermiculite-a promising material for high-temperature heat insulators. Refract Ind Ceram 44:186-193. https://doi.org/10.1023/A:1026312619843

7. Guillot X, Al-Mukhtar M, Bergaya F, Fleureau JM (2002) Porosity assessments in a clay material. C R Geosci 334:105-109. https ://doi.org/10.1016/S1631-0713(02)01705-4

8. Du X, Wu E (2007) Porosity of microporous zeolites A, X and ZSM- 5 studied by small angle $X$-ray scattering and nitrogen adsorption. J Phys Chem Solids 68:1692-1699. https://doi. org/10.1016/j.jpcs.2007.04.013

9. Maitland CF, Buckley CE, O'Connor BH et al (2011) Characterization of the pore structure of metakaolin-derived geopolymers by neutron scattering and electron microscopy. J Appl Crystallogr 44:697-707. https://doi.org/10.1107/S002188981 1021078

10. Iton L, Trouw F, Brun T et al (1992) Small-angle neutron-scattering studies of the template-mediated crystallization of Zsm-5type zeolite. Langmuir 8:1045-1048. https://doi.org/10.1021/ la00040a005

11. Pernyeszi T, Dekany I (2003) Surface fractal and structural properties of layered clay minerals monitored by small-angle X-ray scattering and low-temperature nitrogen adsorption experiments. Colloid Polym Sci 281:73-78. https://doi.org/10.1007/ s00396-002-0758-0

12. Knudsen KD, Fossum JO, Helgesen G, Haakestad MW (2004) Small-angle neutron scattering from a nano-layered synthetic silicate. Physica B 352:247-258. https://doi.org/10.1016/j.physb .2004.07.018

13. Meheust Y, Dagois-Bohy S, Knudsen KD, Fossum JO (2007) Mesoscopic structure of dry-pressed clay samples from small-angle X-ray scattering measurements. J Appl Crystallogr 40:S286S291. https://doi.org/10.1107/S0021889807008552

14. Keller T, Lamandé M, Schjønning P, Dexter AR (2011) Analysis of soil compression curves from uniaxial confined compression tests. Geoderma 163:13-23. https://doi.org/10.1016/j.geode rma.2011.02.006

15. Balima F, Pischedda V, Floch SL et al (2013) An in situ small angle neutron scattering study of expanded graphite under a uniaxial stress. Carbon 57:460-469. https://doi.org/10.1016/j.carbo n.2013.02.019

16. Balima F, Floch SL, San-Miguel A et al (2014) Shear effects on expanded graphite under uniaxial pressure: an in situ small angle neutron scattering study. Carbon 74:54-62. https://doi. org/10.1016/j.carbon.2014.03.002

17. Nguyen AN, Reinert L, Lévêque J-M et al (2013) Preparation and characterization of micron and submicron-sized vermiculite powders by ultrasonic irradiation. Appl Clay Sci 72:9-17. https ://doi.org/10.1016/j.clay.2012.12.007

18. Balima F, Nguyen A-N, Reinert L et al (2015) Effect of the temperature on the structural and textural properties of a 
compressed K-vermiculite. Chem Eng Sci 134:555-562. https:// doi.org/10.1016/j.ces.2015.05.061

19. Nguyen AN, Mirabel L, Duclaux L et al (2015) Room temperature and high temperature sealing properties and compression properties of compressive gaskets made of micrometric vermiculite particles. Appl Clay Sci 114:1-8. https://doi.org/10.1016/j. clay.2015.03.025

20. Le Floch S, Balima F, Pischedda V et al (2015) Small angle scattering methods to study porous materials under high uniaxial strain. Rev Sci Instrum 86:023901. https://doi. org/10.1063/1.4908168

21. Oger PM, Daniel I, Picard A (2006) Development of a low-pressure diamond anvil cell and analytical tools to monitor microbial activities in situ under controlled $\mathrm{P}$ and T. Biochimica et Biophysica Acta (BBA) Proteins Proteomics 1764:434-442. https:// doi.org/10.1016/j.bbapap.2005.11.009

22. Bale H, Schmidt P (1984) Small-angle X-ray-scattering investigation of submicroscopic porosity with fractal properties. Phys Rev Lett 53:596-599. https://doi.org/10.1103/PhysRevLett.53.596

23. Wong P, Howard J (1986) Surface roughening and the fractal nature of rocks. Phys Rev Lett 57:637-640. https://doi. org/10.1103/PhysRevLett.57.637

24. Radlinski AP, Mastalerz M, Hinde AL et al (2004) Application of SAXS and SANS in evaluation of porosity, pore size distribution and surface area of coal. Int J Coal Geol 59:245-271. https://doi. org/10.1016/j.coal.2004.03.002
25. Hurd AJ, Schaefer DW, Smith DM et al (1989) Surface areas of fractally rough particles studied by scattering. Phys Rev B 39:9742-9745. https://doi.org/10.1103/PhysRevB.39.9742

26. Buessem WR, Bartholomew N (1953) The mechanism of the deformation of clay. Clay Clay Miner 2:480-491

27. Graham J, Houlsby G (1983) Anisotropic elasticity of a natural clay. Geotechnique 33:165-180

28. Beaucage G (2012) 2.14—combined small-angle scattering for characterization of hierarchically structured polymer systems over nano-to-micron meter: part II theory. In: Matyjaszewski K, Möller M (eds) Polymer science: a comprehensive reference. Elsevier, Amsterdam, pp 399-409

29. Hammouda B (2010) Analysis of the Beaucage model. J Appl Cryst 43:1474-1478. https://doi.org/10.1107/S00218898100338 56

30. Ruland W (1978) The evaluation of the small-angle scattering of anisotropic lamellar two-phase systems by means of interface distribution functions. Colloid Polym Sci 256:932-936. https:// doi.org/10.1007/BF01383589

Publisher's Note Springer Nature remains neutral with regard to jurisdictional claims in published maps and institutional affiliations. 